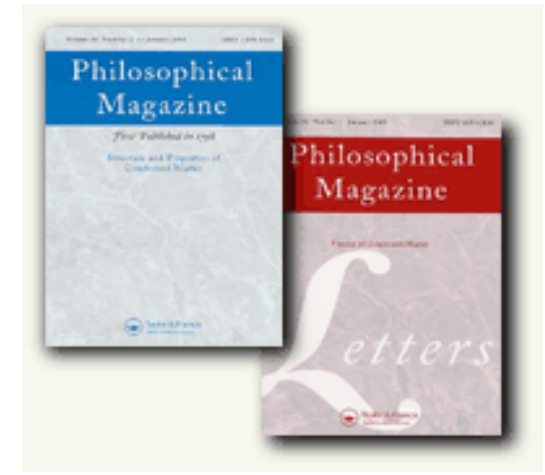

\title{
Growth morphology of single-crystal grains obtained by directional crystallization of an Al-Cu-Fe alloy
}

\begin{tabular}{|r|l|}
\hline Journal: & Philosophical Magazine \& Philosophical Magazine Letters \\
\hline Manuscript ID: & TPHM-10-May-0220.R1 \\
\hline Journal Selection: & Philosophical Magazine \\
\hline Author: & 30 -Aug-2010 \\
\hline Complete List of Authors: & $\begin{array}{l}\text { Surowiec, Marian; Univ. of Silesia, Institute of Material Sc. } \\
\text { Bogdanowicz, Włodzimierz; University of Silesia } \\
\text { Krawczyk, Jacek; University of Silesia } \\
\text { Formanek, Boleslaw; Silesian Univ. of Technology } \\
\text { Sozanska, Maria; Silesian Univ. of Technology }\end{array}$ \\
\hline Keywords: & intermetallic phases, metallic alloys, crystal growth \\
\hline Keywords (user supplied): & $\begin{array}{l}\text { phases in Al-Cu-Fe system, quasicrystal growth morphology, flux } \\
\text { dissolution }\end{array}$ \\
\hline & \multicolumn{2}{l}{} \\
\hline
\end{tabular}

\section{SCHOLARONE Manuscripts}




\title{
Growth morphology of single-crystal grains obtained by directional crystallization of an Al-Cu-Fe alloy
}

\author{
Marian Surowiec $^{1 *}$, Wlodzimierz Bogdanowicz ${ }^{1}$, Jacek Krawczyk ${ }^{1}$, Bolesław Formanek ${ }^{2}$ \\ and Maria Sozanska ${ }^{2}$ \\ ${ }^{1}$ Institute of Material Science, Univ. of Silesia, 40007 Katowice, Poland \\ ${ }^{2}$ Faculty of Materials Engineering and Metallurgy, Silesian Univ. of Technology, \\ 40-019 Katowice, Poland
}

\begin{abstract}
Quasicrystalline as well as crystalline faceted single grains of four phases have been obtained during directional crystallization of an $\mathrm{Al}-\mathrm{Cu}-\mathrm{Fe}$ alloy by the Bridgman technique. The monoclinic $\lambda$ phase $\mathrm{Al}_{13}(\mathrm{Cu}, \mathrm{Fe})_{4}$ dominating at high temperatures formed single-crystal lamellas $0.5 \mathrm{~mm}$ to $1 \mathrm{~mm}$ thick. The second type of attractive morphological forms exhibiting flux dissolution terraces has been observed on spherical single crystals of $\beta$ phase $\mathrm{Al}(\mathrm{Fe}, \mathrm{Cu})$. Rectangular, hexagonal and octagonal shape of dissolution terraces can be seen in the positions of two-, three- and four-fold symmetry axes, respectively. Single quasicrystalline $\psi$ phase $\mathrm{Al}_{6} \mathrm{Cu}_{2} \mathrm{Fe}$ exhibited the icosahedral symmetry with growth forms of a dodecahedron with pentagonal facets. The flux dissolution of $\beta$ phase plays apparently an essential role in a peritectic reaction leading to quasicrystalline $\psi$ phase formation. Polygonal single grains of $\omega$ phase $\mathrm{Al}_{7} \mathrm{Cu}_{2} \mathrm{Fe}$ exhibiting the tetragonal symmetry formed the fourth type of thermodynamically stable growth forms. Single grains of the $\omega$ phase crystallized in a form of pellets with an octagonal cross-section. The growth morphology of stable phases has been investigated by the scanning electron microscopy. The chemical composition of the growth forms described has been confirmed by the X-ray microanalysis using a scanning electron microscope whereas the phase composition has been determined by the electron selected area diffraction and X-ray powder diffraction.
\end{abstract}

Keywords: phases in Al-Cu-Fe system, quasicrystal growth morphology, flux dissolution.

\section{$\S 1$. Introduction}

Investigations of the phase composition of $\mathrm{Al}-\mathrm{Cu}-\mathrm{Fe}$ system lead to working out reliable and detailed phase diagrams [1-6] defining conditions for crystallization of the quasicrystalline icosahedral stable phase. Rapid solidification as well as normal casting process of the alloy combined with thermal annealing and subsequent quenching were commonly used [7-12]. The icosahedral $\psi$ phase $\mathrm{Al}_{6} \mathrm{Cu}_{2} \mathrm{Fe}$ has common equilibrium phase fields with three phases at $800{ }^{\circ} \mathrm{C}\left(\beta, \lambda\right.$ and liquid), at $700-720{ }^{\circ} \mathrm{C}$ the equilibrium field covers four phases $\left(\beta, \lambda, \omega\right.$ and liquid) and at $680^{\circ} \mathrm{C}$ the $\psi$ phase is in equilibrium with $\beta, \lambda$ and $\omega$ phases [2]. At $680{ }^{\circ} \mathrm{C}$ the perfectly stable icosahedral quasicrystalline $\psi$ phase occurs in the range of compositional triangle $\mathrm{Al}_{62.4} \mathrm{Cu}_{24.4} \mathrm{Fe}_{13.2}, \mathrm{Al}_{65} \mathrm{Cu}_{23} \mathrm{Fe}_{12}$ and $\mathrm{Al}_{61} \mathrm{Cu}_{28.4} \mathrm{Fe}_{10.6}$ [5]. The $\beta$ phase $\mathrm{Al}(\mathrm{Cu}, \mathrm{Fe})$ ( $\mathrm{Pm} 3 \mathrm{~m}$ space group) has a $\mathrm{CsCl}$ type structure, the monoclinic dominant $\lambda$ phase $\mathrm{Al}_{13}(\mathrm{Cu}, \mathrm{Fe})_{4}$ is described by $\mathrm{C} 2 / \mathrm{m}$ space group and the $\omega$ phase $\mathrm{Al}_{7} \mathrm{Cu}_{2} \mathrm{Fe}$ (P4/mnc space group) exhibits the tetragonal symmetry. The structural characteristic of the phases has been reported earlier by Liu and Köster [13] and by Gigla et al. [14].

*) Corresponding Author

e-mail: marian.surowiec@us.edu.pl

Telephone: +48323591855

Fax: +48 323592133 
Millimetre sizes of Al-Cu-Fe alloy icosahedral single grains have been obtained by annealing at $1095 \mathrm{~K}$ for a few dozen hours and followed by immediate quenching carried out by Ishimasa and Mori [8]. Large single grains of the quasicrystalline icosahedral $\mathrm{Al}-\mathrm{Cu}-\mathrm{Fe} \psi$ phase have been prepared by multiple cyclic heat treatments by Lograsso and Danley [12].

The most evident form of thermodynamically stable process of quasicrystals crystallization was confirmed by the polyhedral faceted growth morphology. The flux growth technique was successfully applied by Fisher et al. [15] for growth of faceted large singlegrain quasicrystals from high-temperature metallic solutions. The authors used decanting technique to grow icosahedral quasicrystals of single grain alloys of $\mathrm{Ho}-\mathrm{Mg}-\mathrm{Zn}, \mathrm{Al}-\mathrm{Pd}-\mathrm{Mn}$ and Al-Ga-Pd-Mn exhibiting the dodecahedral morphology as well as for the growth of decagonal Al-Ni-Co and Al-Pd-Mn approximate single grains distinguished by decaprismatic columns (ten prism faces with the 10-fold axis as the rotation axis).

The paper reports on the growth equilibrium morphology of both quasicrystalline as well as periodic phases obtained during a directional crystallization of an $\mathrm{Al}-\mathrm{Cu}-\mathrm{Fe}$ alloy for a chemical composition close to the above mentioned compositional triangle of the $\psi$ phase occurrence. The phases crystallized as polygonal single crystals. A single-grain morphology of four phases crystallized in the same ingot will be described: a monoclinic dominant $\lambda$ phase, belonging to the cubic system $\beta$ phase, quasicrystalline phase $\psi$ and periodic phase $\omega$ with tetragonal symmetry. In the case of $\beta$ phase the flux dissolution morphology is described.

\section{$\S 2$. Experimental}

Polyhedral equilibrium phases were formed in ingots of an $\mathrm{Al}-\mathrm{Cu}-\mathrm{Fe}$ alloy, for which the nominal composition was 65 at. $\% \mathrm{Al}, 20$ at.\% $\mathrm{Cu}$ and 15 at.\% Fe. The ingots of total mass of approximately $40 \mathrm{~g}, 15 \mathrm{~mm}$ in diameter and $60 \mathrm{~mm}$ high, were sharpened at the bottom. The specimens were synthesized in a helium atmosphere inside alumina crucibles starting from $99.997 \%$ pure $\mathrm{Al}, 99.9 \%$ pure $\mathrm{Cu}$ and $99.98 \% \mathrm{Fe}$ using the BridgmanCzochralski-Growth (BCG) apparatus equipped with an induction furnace. The initial temperature of the melt was increased up to $1550{ }^{\circ} \mathrm{C}$ and the crystallization rate during Bridgman directional crystallization was $6 \mathrm{~mm} / \mathrm{h}$ resulting in an approximate cooling rate of $1.3^{\circ} \mathrm{C} / \mathrm{min}$. Single crystal grains have crystallized in the melt or were subject to the flux dissolution until the melt has been removed by the solidification shrinkage. In our experiment the samples were not quenched in order to prevent a structural transformation from the icosahedral phase to non-icosahedral approximate structure. The ingot was crushed into several pieces and different growth forms previously closed inside cavities and pores were subject to the investigation of growth morphology by the scanning electron microscopy (SEM) using primary and secondary electrons. The selected area diffraction (SAD) as well as $\mathrm{X}$-ray powder diffraction confirmed the phase identification of the predicted phase composition in the Al-Cu-Fe system. Positions of X-ray diffraction lines were recalculated into interplanar distances.

\section{$\S 3$. Results}

Four faceted morphology forms have been found inside cavities: monoclinic $\lambda$ phase grown in a shape of lamellas with a rhomboidal cross section, terraced forms of icosahedral $\beta$ phase revealing symmetry elements, dodecahedrons with pentagonal facets of icosahedral $\psi$ phase and octagonal pellets of $\omega$ phase.

\subsection{Growth morphology of the $\lambda$ phase}


The first type of faceted single crystals was formed inside the ingot by the dominant monoclinic $\lambda$ phase $\mathrm{Al}_{13}(\mathrm{Cu}, \mathrm{Fe})_{4}(\mathrm{C} 2 / \mathrm{m}$ space group). Growth morphology forms observed inside cavities revealed lamellas up to $50 \mu \mathrm{m}$ thick, terminated by smooth (010) crystal facets in a shape of rhombi (Fig. (1)). A fragment of lamella is also seen in the lower part of Fig. 2. The characteristic angle $\beta=108^{\circ}$ for the monoclinic $\lambda$ phase structure is marked in Fig. 1.

\subsection{Flux dissolution morphology of the $\beta$ phase}

The second type of attractive morphological forms observed inside shrinkage cavities were spherical single grains of $\beta$ phase $\mathrm{Al}(\mathrm{Fe}, \mathrm{Cu})(\mathrm{Pm} 3 \mathrm{~m}$ space group, $a=0.29076 \mathrm{~nm})$ [14]. The solidified colonies of droplets of $\beta$ phase exhibit apparently flux dissolution terraces (Fig. 2). Positions of threefold symmetry axes have the same orientation in all spherical single grains (Fig. 2). Rectangular, hexagonal and octagonal shape of the dissolution terraces can be seen in the positions of two-, three- and four-fold symmetry axes, respectively (Fig 3 (a) and (b)). Therefore flat steps of terraces are parallel to $\{110\},\{111\}$ and $\{100\}$ crystallographic planes, respectively. Spatial positions of symmetry elements observed on droplets of the $\beta$ phase correspond to the distribution of symmetry elements in the cube (Fig. 3). The flux dissolution of the surface of $\beta$ phase revealed (111) planes inclined to the surface (Fig. 4) as well as terraced hollows with an amphitheatric architecture (marked as A in Fig. 4). The width of terraces extends up to $12 \mu \mathrm{m}$ whereas the height of terrace steps is less than $1 \mu \mathrm{m}$. Single crystal droplets of the $\beta$ phase existed at the very initial stage of flux dissolution (Fig. 5).

\subsection{Morphology of Al-Cu-Fe single quasicrystals}

Single quasicrystals of $\psi$ phase $\mathrm{Al}_{6} \mathrm{Cu}_{2} \mathrm{Fe}$ exhibited the icosahedral symmetry with dodecahedral growth forms - 12 faces perpendicular to the 5-fold axes (Fig. 6). The symmetry was confirmed by the electron diffraction (see the insert in Fig. 6a). The dodecahedra have irregularities and different dimensions of edges extending up to $160 \mu \mathrm{m}$. The faces of dodecahedra are not perfectly smooth and emerge from the residual flux (Fig. 6 (b)).

\subsection{Growth forms of the $\omega$ phase}

Polygonal single grains of $\omega$ phase $\mathrm{Al}_{7} \mathrm{Cu}_{2} \mathrm{Fe}(\mathrm{P} 4 / \mathrm{mnc}, a=0.6336 \mathrm{~nm}, c=1.482 \mathrm{~nm})$ exhibiting tetragonal symmetry form randomly distributed pellets between terraced growth forms of the $\beta$ phase (Fig. 7). In general the surfaces of pellets are not smooth, however an octagonal cross section can be observed (the insert in Fig. 7). The pellets exhibit eight lateral facets, four of $\{100\}$ type and four between them of $\{110\}$ type. The tetragonal prisms are about $50 \mu \mathrm{m}$ in diameter and $60 \mu \mathrm{m}$ high.

\subsection{Phase identification}

The selected area diffraction (e.g. inserts in Fig. 1 and Fig. 6 (a)) and X-ray powder diffraction confirmed the inherence of all phases described above. Positions of X-ray diffraction lines were recalculated into interplanar distances. The lattice parameter of the $\beta$ phase was determined as $a=0.29076 \mathrm{~nm}$, for the $\lambda$ phase $a=1.5849 \mathrm{~nm}, b=0.8083 \mathrm{~nm}$ and $c=1.2476 \mathrm{~nm}, \beta=107.7^{\circ}$ and for the $\omega$ phase $a=0.6336$ and $c=1.482 \mathrm{~nm}$.

\section{$\S 4$. Discussion and conclusion}

Faceted single crystals of three crystalline phases: monoclinic $\lambda$, cubic $\beta$ and tetragonal $\omega$ surround the polygonal single units of quasicrystalline phase $\psi$. The melting point of the $\lambda$ and $\beta$ phase is above $1100{ }^{\circ} \mathrm{C}$. Both $\psi$ and $\omega$ phase are formed during peritectic reactions in the temperature range $700-872{ }^{\circ} \mathrm{C}$ and $700-740{ }^{\circ} \mathrm{C}$, respectively [16]. Therefore four 
phases can coexist in the overlapping temperature area of $700-740{ }^{\circ} \mathrm{C}$. In our experiment the overheating of the melt directly before the directional crystallization was a favourable factor for pores and cavities formation - the melt temperature was increased up to $1550{ }^{\circ} \mathrm{C}$. Gas bubbles as well the material shrinkage during cooling provided a helpful micro-space for the morphology visualization of single crystal units after the ingot crushing.

The main result of the paper is related to the flux dissolution of the high temperature $\beta$ phase which apparently provides necessary material for peritectic formation of quasicrystalline phase $\psi$. We assume that solid droplets of the $\beta$ phase, formed primary by the surface tension of liquid in a space of a bubble or shrinkage, may have a significant contribution during the flux dissolution (Fig. 4 and 5) to the peritectic reaction $\beta+\lambda+\mathrm{L} \rightarrow \psi$ identified e.g. by Lograsso and Delaney [12]. The $\beta$ phase consumption by dissolution was confirmed during investigations of phase transformations of $\mathrm{AlCuFe}$ alloys performed by Zhang et al. [10]. An attractive flux dissolution morphology of cubic $\beta$ phase confirms a high structural perfection of the primary crystallized droplet-like single crystals. Note that all ten arrowed threefold axes (Fig. 4) have the same spatial orientation, therefore all the colony of droplets forms apparently one single crystal. The flux dissolution morphology of the $\beta$ phase reveals three kinds of crystallographic planes $\{111\},\{100\}$ and $\{110\}$. The flux dissolution of $\beta$ phase droplets follows from chemical potential differences between the melt and the solid phase.

The tetragonal $\omega$ phase was formed in the smallest amount in the ingot apparently by an analogous peritectic reaction $\lambda+\mathrm{L} \rightarrow \omega$ [17].

The quasicrystals of face centred icosahedral $\mathrm{Al}-\mathrm{Cu}-\mathrm{Fe}$ grow with dodecahedral morphology exhibiting 12 faces perpendicular to the 5-fold axes. Some of as grown facets were decorated in a similar way as fivefold facets of Al-Pd-Mn quasicrystals found by Beeli and Nissen [18]. The results concerning the decorations will be published elsewhere.

The shape of different observed single grains confirms our SAD and X-ray powder diffraction phase identification. The four phases detected indicate that sample was not in a thermodynamic equilibrium. However, the observed stable growth forms can be explained in relation to Scheil-Gulliver approach of non-equilibrium process of solidification. This approach approximates a non-equilibrium solidification by assuming a local equilibrium of the advancing solidification front at the solid-liquid interface [19].

\section{References}

1. A. J. Bradley, H.J. Goldschmidt, J. Inst. Met. 62403 (1939).

2. F.W. Gayle, A.J. Shapiro, F.S. Biancanello, W. J. Böttinger, Metall. Trans. A23 2409 (1992).

3. A.P. Prevarskyi, Russ. Met. 4154 (1971).

4. L.M. Zhang, R. Lueck, Z. Metallk. 94774 (2003).

5. D. Gratias, Y. Calvayrac, J. Devaud-Rzepski, F. Faudot, M. Harmelin, A. Quivy and P.A. Bancel, J. Non-Cryst. Solids, 153-154 482 (1993).

6. C. P. Wang, X. J. Liu, L. Zhang and K. Ishida, Landolt - Börnstein New Series, IV/11A2, Springer 2005, p. 1.

7. A. P. Tsai, A. Inoue and T. Masumoto, J. Mater. Sci. Lett. 61403 (1987).

8. T. Ishimasa and M. Mori, Phil. Mag. Lett. 62357 (1990).

9. M. Asahi, T. Maki, T. Kishide and K. Tatsugawa, J. Non-Cryst. Solids, 156-158 923

(1993).

10. L. M. Zhang, J. Schneider and R. Lück, Intermetallics, 131195 (2005).

11. 15. G. Rosas, J. Reys-Gasga and R. Perez, Mat. Charact. 58765 (2007).

12. T. A. Lograsso and D.W. Delaney, J. Mater. Res., 112125 (1996). 
13. W. Liu and U. Köster, Z. Metallkunde, 82791 (1991).

14. M. Gigla, J. Lelatko and M. Surowiec, Applied Crystallography, Proc. XVII Conference (Wisla, Poland, 31 August - 4 September 1997) edited by H. Morawiec, and D. Stroz, (Singapore: World Scientific 1998), p. 55.,

15. I. R. Fisher, M. J. Kramer, Z. Islam, T. A. Wiener, A. Kracher, A. R. Ross, T. A. Lograsso, A. I. Goldman and P. C. Canfield, Mat. Sci. Eng., 294-296 10 (2000).

16. P.A. Bancel, Quasicrystals: The state of art, edited by D. P. DiVincenzo and P. J. Steinhard, (Singapore: World Scientific 1998), p. 26.

17. F. Faudot, A. Quivy, Y. Calvayrac, D. Gratias, M. Harmelin, Mat. Sc. Eng., A133 383 (1991)

18. C. Beeli and H.-U. Nissen, Phil. Mag. B, 68487 (1993).

19. E. Scheil, Z. Metallk., 3470 (1942).

Figure captions (Caution: major reduction of pictures size on Fig. 2 - Fig. 5 will make photos useless)

Fig. 1. Morphology of single crystal monoclinic $\lambda$ phase; visible rhombi are (010) planes. Characteristic angle $\beta=108^{\circ}$ is marked. Inserts: the selected area diffraction pattern and a fragment of single lamella of $\lambda$ phase.

Fig. 2. Single crystal droplets of the $\beta$ phase $\mathrm{Al}(\mathrm{Fe}, \mathrm{Cu})$. Arrows show positions of threefold symmetry axes revealed apparently by the flux dissolution.

Fig 3. Spatial positions of 2-, 3- and 4-fold symmetry axes revealed on terraced single crystal droplets of the $\beta$ phase.

Fig. 4. Flux dissolution morphology of the surface of the $\beta$ phase; terraces are formed by (111) crystallographic planes which are inclined to the surface. A - a hollow with an amphitheatric architecture.

Fig. 5. Flux dissolution initiation of terraces on spherical droplets of the $\beta$ phase.

Fig. 6. Pentagonal dodecahedra of quasicrystalline $\psi$ phase $\mathrm{Al}_{6} \mathrm{Cu}_{2} \mathrm{Fe}$. (a) Pentagonal faces of a single grain. (b) Solidified residual flux covering dodecahedra. Insert in (a) shows an electron diffraction pattern of icosahedral $\psi$ phase.

Fig. 7. Randomly distributed pellets of the $\omega$ phase $\mathrm{Al}_{7} \mathrm{Cu}_{2} \mathrm{Fe}$ between terraced growth forms of the $\beta$ phase. The insert reveals octagonal cross section of a single pellet. 


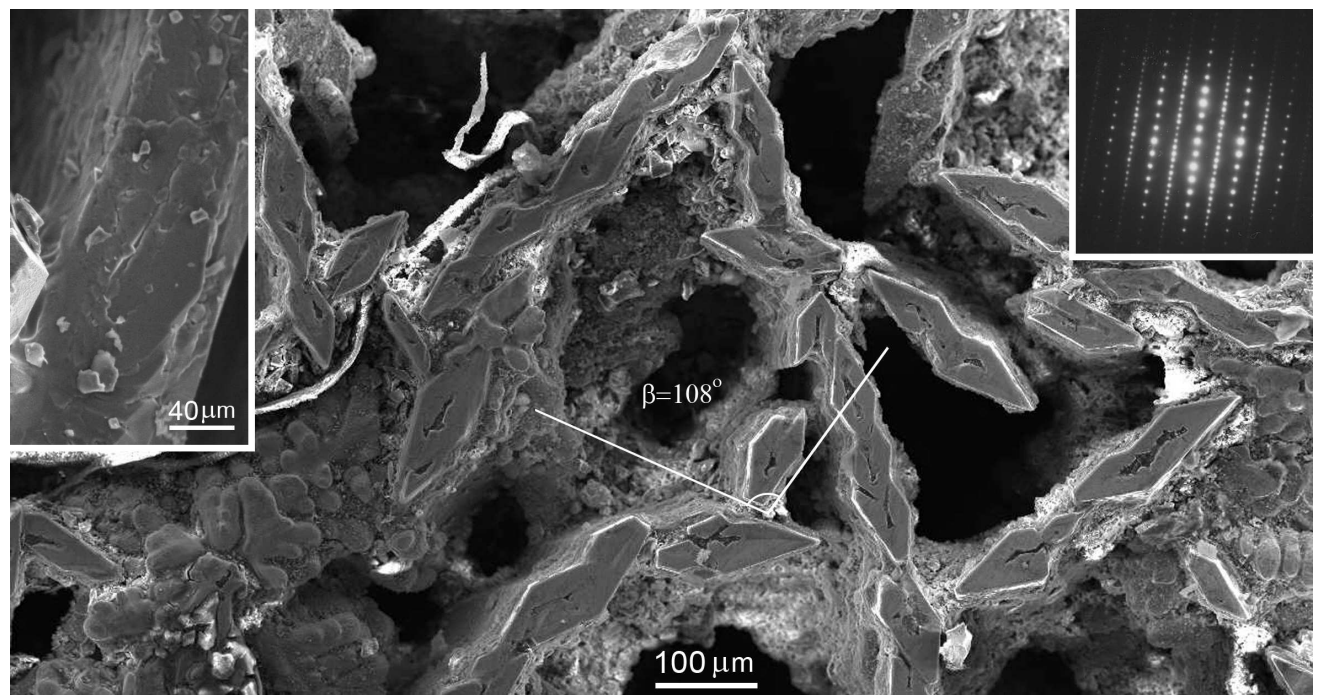

Fig.1. Morphology of single crystal monoclinic $\lambda$ phase; visible rhombi are (010) planes. Characteristic angle $\beta=108^{\circ}$ is marked. Inserts: the selected area diffraction pattern and a fragment of single lamella of $\lambda$ phase. $199 \times 105 \mathrm{~mm}(300 \times 300$ DPI $)$ 


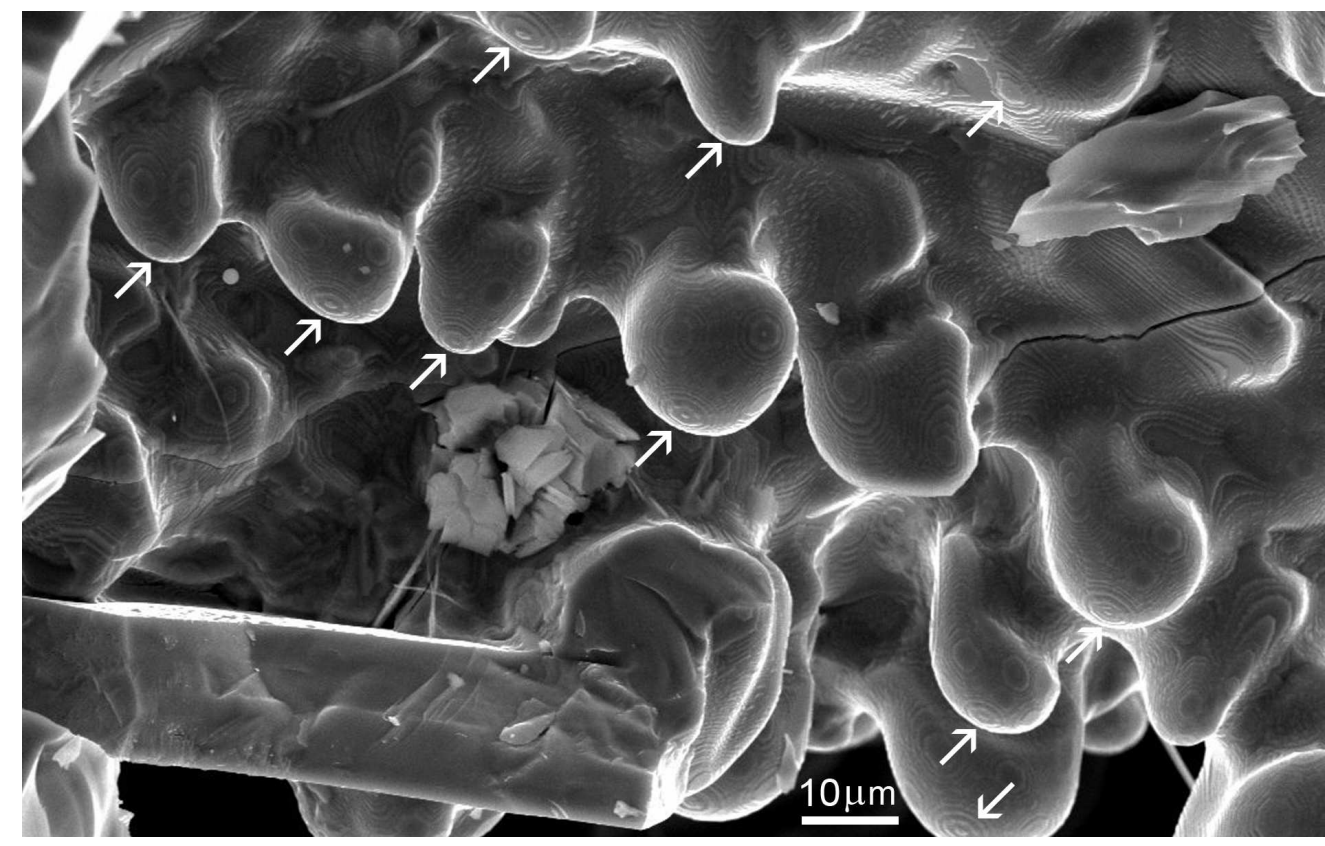

Fig. 2. Single crystal droplets of the $\beta$ phase $\mathrm{Al}(\mathrm{Fe}, \mathrm{Cu})$. Arrows show positions of threefold symmetry axes revealed apparently by the flux dissolution. $160 \times 101 \mathrm{~mm}(300 \times 300 \mathrm{DPI})$ 

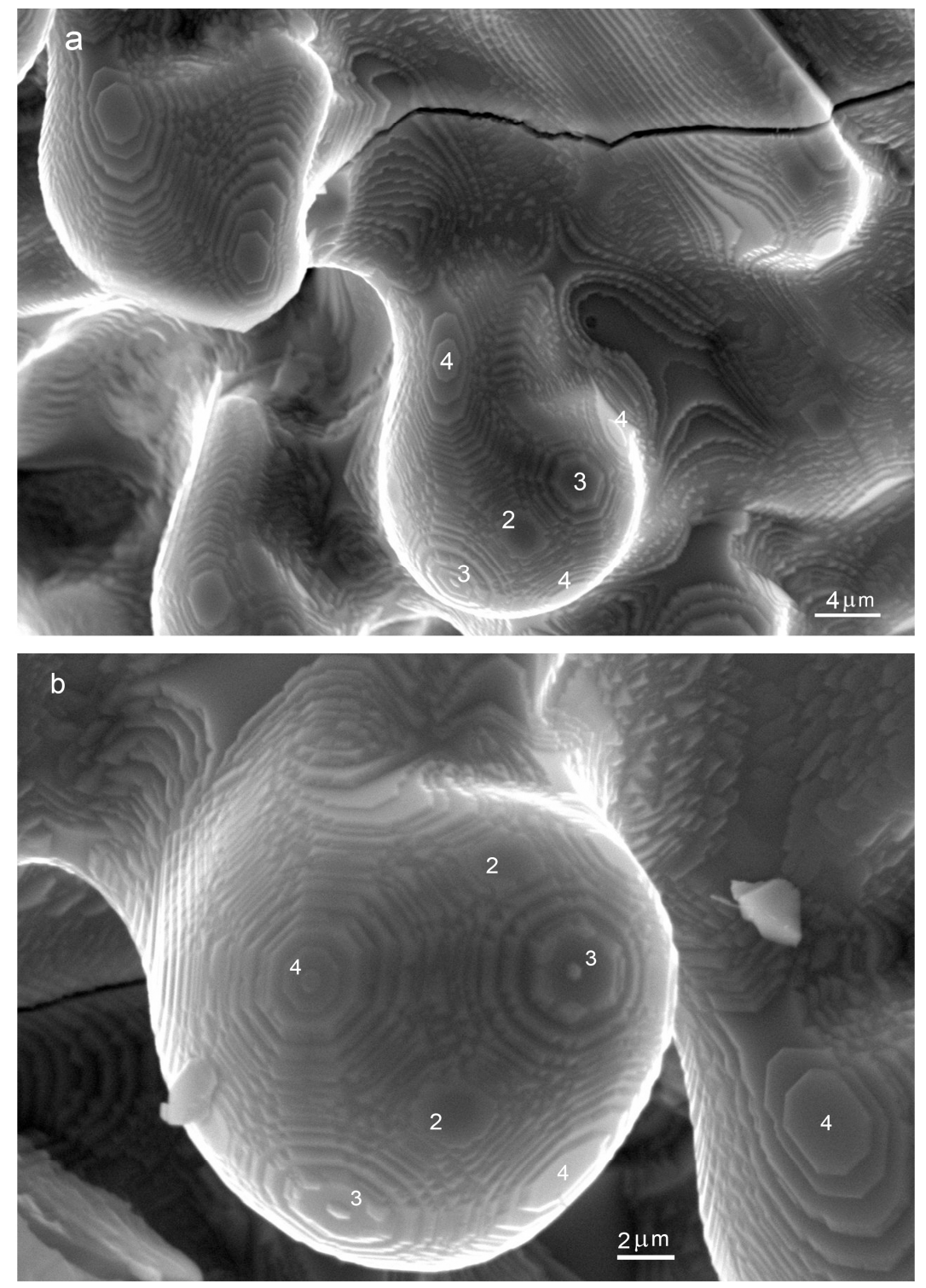

Fig. 3. Spatial positions of 2-, 3- and 4-fold symmetry axes revealed on terraced single crystal droplets of the $\beta$ phase. $209 \times 297 \mathrm{~mm}(300 \times 300 \mathrm{DPI})$ 
Fig. 4. Flux dissolution morphology of the surface of the $\beta$ phase; terraces are formed by (111) crystallographic planes which are inclined to the surface. A - a hollow with an amphitheatric architecture. $199 \times 104 \mathrm{~mm}(300 \times 300$ DPI $)$ 


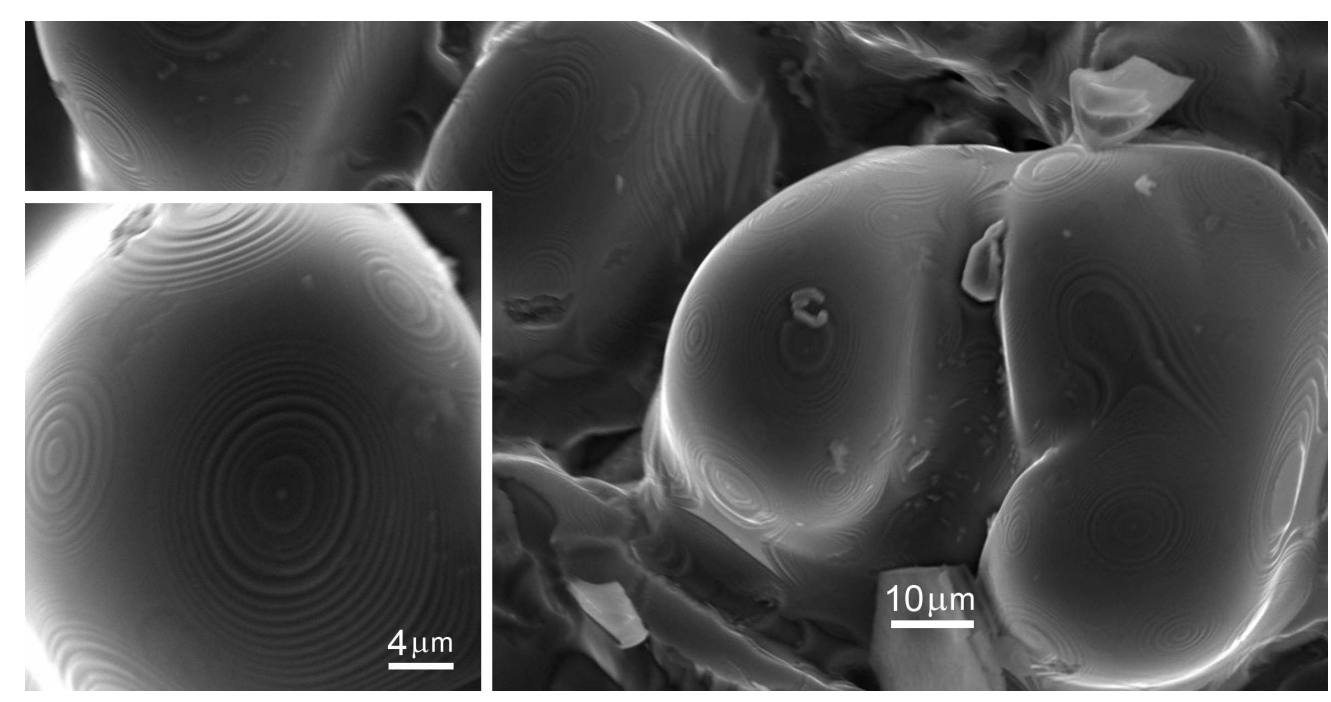

Fig. 5. Flux dissolution initiation of terraces on spherical droplets of the $\beta$ phase. $199 \times 102 \mathrm{~mm}(300 \times 300$ DPI $)$ 

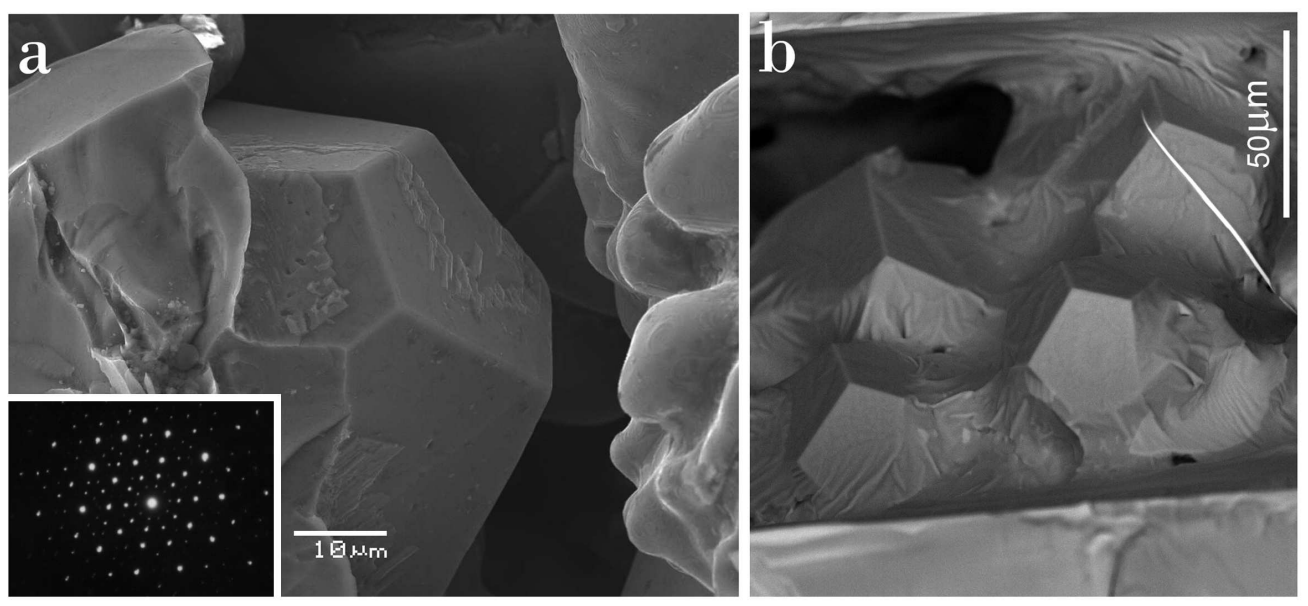

Fig. 6. Pentagonal dodecahedra of quasicrystalline $\psi$ phase Al6Cu2Fe. (a) Pentagonal faces of a single grain. (b) Solidified residual flux covering dodecahedra. Insert in (a) shows an electron diffraction pattern of icosahedral $\psi$ phase. $199 \times 91 \mathrm{~mm}(300 \times 300 \mathrm{DPI})$ 


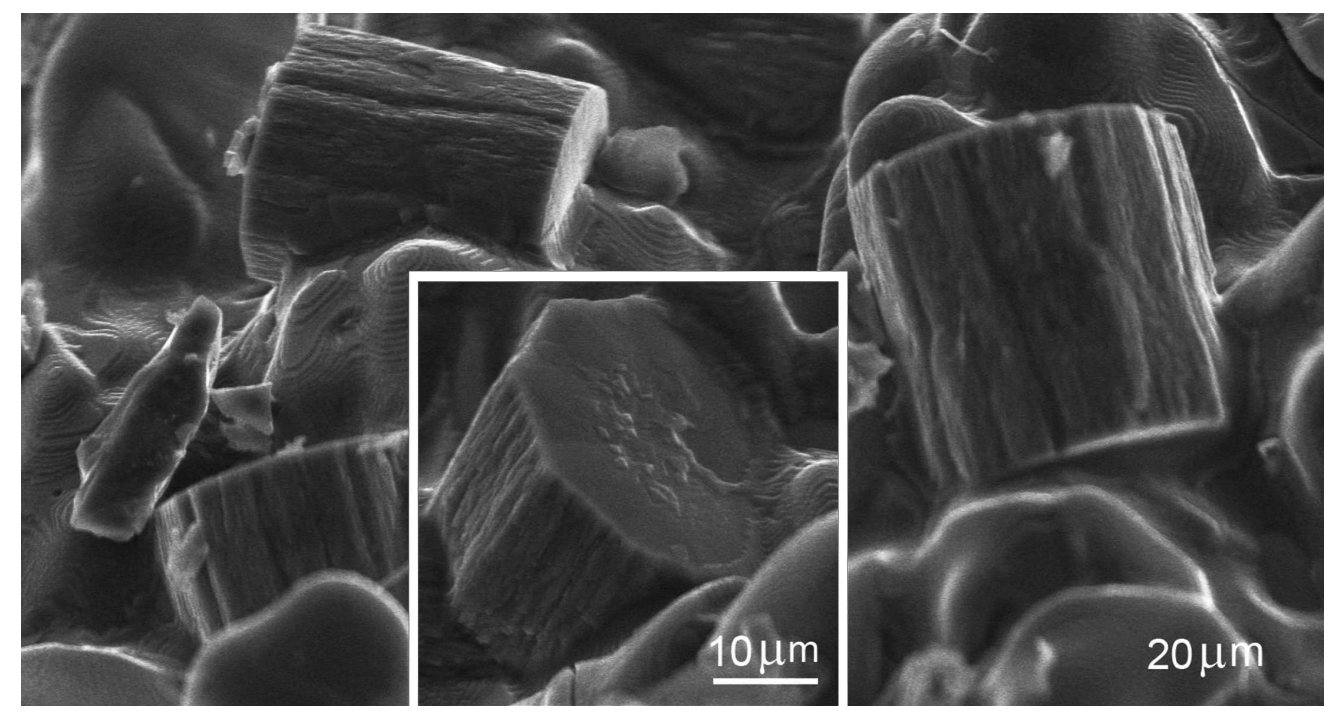

Fig. 7. Randomly distributed pellets of the $\omega$ phase Al7Cu2Fe between terraced growth forms of the $\beta$ phase. The insert reveals octagonal cross section of a single pellet. $187 \times 99 \mathrm{~mm}(300 \times 300$ DPI $)$ 